\title{
Electrostatic repulsion regulates hiv-I capsid hexamer plasticity and assembling capacities into core-like structures
} Sonia Brun*1,2,3, Laurent Chaloin 1,2,3, Bernard Gay 1,2,3, Eric Bernard 1,2,3, Christian Devaux $1,2,3$, Corinne Lionne ${ }^{1,2,3}$, Nathalie Chazal ${ }^{1,2,3}$ and Laurence Briant ${ }^{1,2,3}$

Address: ${ }^{1}$ Université Montpellier 1, Centre d'études d'agents Pathogènes et Biotechnologies pour la Santé (CPBS), France, ${ }^{2} \mathrm{CNRS}, \mathrm{UMR} 5236$, CPBS, F-34965 Montpellier, France and ${ }^{3}$ Université Montpellier 2, CPBS, F-34095 Montpellier, France

* Corresponding author

from Frontiers of Retrovirology: Complex retroviruses, retroelements and their hosts

Montpellier, France. 21-23 September 2009

Published: 24 September 2009

Retrovirology 2009, 6(Suppl 2):P30 doi:10.1186/1742-4690-6-S2-P30

This abstract is available from: http://www.retrovirology.com/content/6/S2/P30

(c) 2009 Brun et al; licensee BioMed Central Ltd.

Capsid protein (CA) is the major component of the HIV1 core. Three major phosphorylation sites have been identified at positions $\mathrm{S}_{109}, \mathrm{~S}_{149}$ and $\mathrm{S}_{178}$ in amino-acid sequence of CA. Here we investigated the possible consequences of phosphorylation at these sites on CA hexamer formation and core assembly. Using in silico modeling approaches we found that the presence of a phosphate group at each position impacts CA hexamer organization and flexibility. The biological relevance of molecular modeling was evaluated analyzing in vitro assembly properties of bacterially expressed CA bearing $S_{109} D, S_{149} D$ or $\mathrm{S}_{178} \mathrm{D}$ substitutions that mimic constitutive phosphorylation at these sites. Constitutive negative charge at positions 109, 149 but not at position 178 impaired in vitro assembly of mature CA. In vivo, the corresponding mutations were found to impact the core morphology of HIV1 mutants bearing the corresponding mutation. Moreover, these viruses lacked infectivity since they were unable to produce proviral DNA. Altogether our data indicate that phosphorylation may impact structural properties of CA hexamers and of HIV-1 cores. Its contribution in HIV1 replication appears to play an important role in HIV-1 life cycle. 